\title{
ASTRONOMICAL MODELS OF PAHS AND DUST
}

\author{
B.T. Draine ${ }^{1}$
}

\begin{abstract}
Physical dust models which include a PAH component are quite successful in reproducing the measured extinction $v s$. wavelength in the Milky Way, the infrared (IR) emission observed from Milky Way regions and other galaxies, and a number of other observational constraints. Many of the adopted PAH properties are necessarily highly idealized.

The observed variations in the $7.7 \mu \mathrm{m} / 11.3 \mu \mathrm{m}$ band ratio can be reproduced by varying the PAH ionization balance. Changing the spectrum of the starlight heating the PAHs affects the overall strength of the PAH emission (relative to total IR), but has relatively little effect on the $7.7 \mu \mathrm{m} / 11.3 \mu \mathrm{m}$ band ratio.
\end{abstract}

\section{Introduction}

PAHs are an important component of the dust population. They make a substantial contribution to the overall extinction by dust, accounting for $\sim 20 \%$ of the total IR power from a star-forming galaxy like the Milky Way. See Tielens (2008) for a general review of PAHs.

Physical models for dust, including a PAH population, have been developed (Desert et al. 1990; Weingartner \& Draine 2001; Li \& Draine 2001a; Zubko et al. 2004; Draine \& Li 2007; Draine \& Fraisse 2009; Compiegne et al. 2010). For application to the Milky Way, these models are subject to an array of observational constraints:

- Starlight extinction: The observed extinction of starlight strongly constrains the dust size distribution and composition. Extinction curves have been measured using stars in the Milky Way (MW), Large Magellanic Cloud (LMC), and Small Magellanic Cloud (SMC), and in a limited number of other galaxies using background quasistellar objects (QSOs) and gamma ray burst (GRB) afterglows as the light source. The extinction is best-studied

1 Dept. of Astrophysical Sciences, Princeton University 
in the MW, where spectral features at $10 \mu \mathrm{m}$ and $18 \mu \mathrm{m}$ show that amorphous silicates are a major constituent, and a strong extinction "bump" near $2175 \AA$ is usually interpreted as due to $s p^{2}$-bonded carbon, as in graphite or PAHs.

- Starlight polarization: The extinction is polarization-dependent, requiring that some of the grains be nonspherical and aligned with respect to the Galactic magnetic field.

- Scattering of starlight: A substantial fraction of the observed extinction in the optical is due to scattering, requiring that some of the grains must be large enough to efficiently scatter optical light.

- Abundance constraints: The grain model should incorporate elements only as allowed by the total abundance of the element minus the fraction observed to be in the gas phase.

- Infrared emission: The dust model should reproduce the IR emission spectra observed from regions with different intensities of starlight heating the dust.

- X-Ray scattering: The dust composition and size distribution must be such as to reproduce the observed strength and angular distribution of X-ray scattering by interstellar dust.

- Microwave emission: Dust-correlated microwave emission is attributed to rotational emission from very small dust grains (Draine \& Lazarian 1998a,b). This constrains the abundances of the smallest grains.

- Dust in meteorites: Presolar grains with isotopic anomalies are found in meteorites. These were part of the interstellar grain population 4.6 Gyr ago, when the solar system formed.

Models for MW dust are therefore strongly constrained (see Draine 2003, for further discussion).

\section{Physical models for dust with PAHs}

The Infrared Space Observatory (ISO) discovered, and Spitzer Space Telescope confirmed, that strong PAH emission features are routinely present in the IR spectra of star-forming galaxies. The relative strengths of the PAH features do vary somewhat from galaxy to galaxy, but in fact show surprising "universality".

Measured extinction curves in the MW, LMC, and SMC have many similarities: (1) A general trend to rise strongly from the near-IR $(\lambda \approx 1 \mu \mathrm{m})$ to the FUV $(\lambda \approx 0.1 \mu \mathrm{m})$. (2) A tendency for $A_{V} / N_{\mathrm{H}}$ to vary in proportion to metallicity the MW, LMC, and SMC all seem to have similar fractions of "refractory elements" in dust. Models to reproduce the observed $A_{\lambda} / N_{\mathrm{H}}$ require a substantial fraction of abundant refractory elements (C, Mg, $\mathrm{Si}, \mathrm{Fe})$. 
However, significant regional variations in the shape of the extinction curve are observed. The function $A_{\lambda} / A_{V}$ varies from sightline-to-sightline within the MW, and within the LMC and SMC. In particular, the relative strength of the $2175 \AA$ feature decreases as one goes from MW to LMC to SMC. A physical grain model should be able to accomodate these variations.

Contemporary dust models for dust in the MW (and other galaxies) (e.g., Weingartner \& Draine 2001; Li \& Draine 2001b; Zubko et al. 2004; Draine \& Li 2007; Draine \& Fraisse 2009) have amorphous silicate and carbonaceous material as the principal dust materials. The specific mix of carbonaceous material (PAH, graphite, various forms of amorphous carbon...) varies from model to model.

A dust model consists of specifying the grain composition and size distribution. The MW, LMC, and SMC extinction curves can be reproduced by models consisting of amorphous silicate + graphite + PAHs, with only changes in the size distributions (and relative abundances) of the 3 components from sightline to sightline (Weingartner \& Draine 2001). In galaxies lacking measured extinction curves, it is reasonable to use the size distribution obtained for the MW unless we are forced to change some property, such as the relative abundance of PAHs.

For each grain composition and size, we require scattering and absorption cross sections as a function of wavelength. For grains with radii $a>100 \AA$, we calculate the scattering and absorption cross sections by solving Maxwell's equations for the appropriate grain size and dielectric function, usually assuming either a spherical or spheroidal shape (Draine \& Fraisse 2009). To calculate the response to singlephoton heating, we also require the heat capacity of each grain.

The PAHs are small enough that scattering is expected to be unimportant, and are also small enough that calculating absorption cross sections using "bulk" optical constants - even if these were available - may be a poor approximation. Instead, we rely on experimental and theoretical studies of PAH absorption cross sections per C atom. The PAH opacity adopted by Draine \& Li (2007, hereafter DL07) is shown in Figure 1.

Our knowledge of absorption cross sections for PAHs (neutrals and ions) is limited, especially as regards large species ( $c f$. http://astrochemistry.ca.astro. it/database/pahs.html). At this time most dust models make the simplifying assumption that the band profiles for PAHs are "universal". Band positions, widths, and strengths are adopted that appear to be consistent with (1) astronomical observations of band profiles, and (2) laboratory and theoretical studies (to the extent available). In reality, cross sections vary significantly from PAH to PAH, and from region to region (see Peeters 2011, in this volume) but we are not yet in a position to include this in models. Ionization matters: the adopted band strengths depend on whether the PAH is neutral or ionized. A first study on the role of charge state of PAHs in ultraviolet extinction has been recently performed (Cecchi-Pestellini et al. 2008; see Mulas et al. 2011 in this volume). 

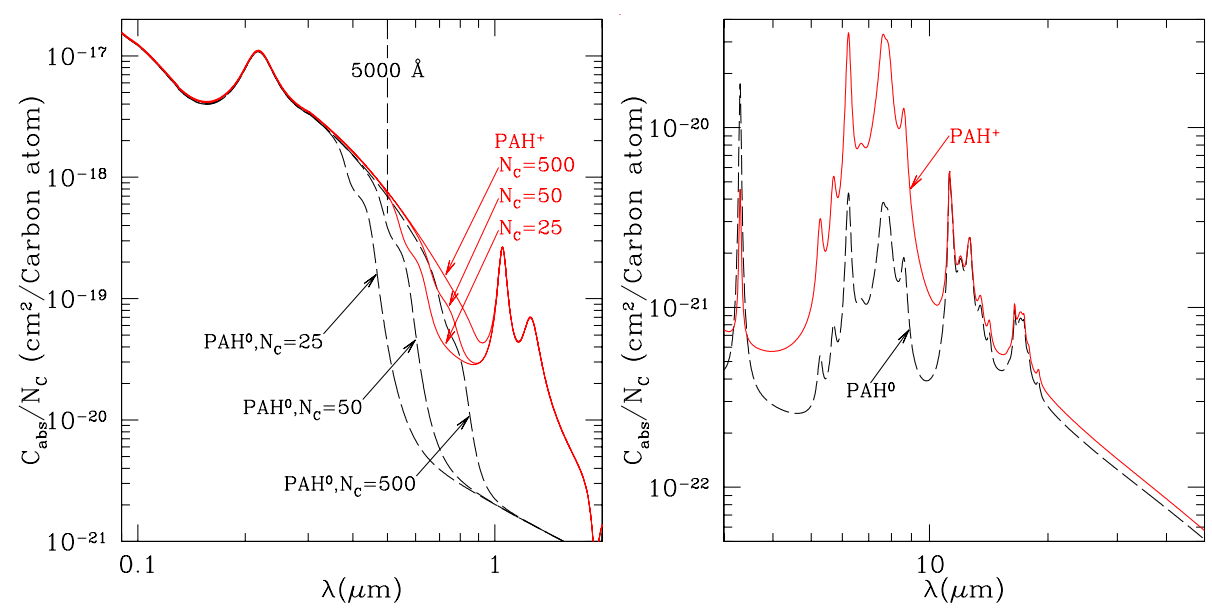

Fig. 1. Radiative cross sections adopted for PAHs (neutral and cations) in the UV-optical (left) and IR (right). After Draine \& Li (2007).

\section{Modeling the IR emission}

In the ISM, grains and PAHs are heated primarily by absorption of starlight photons, and cooled by emission of IR photons. Radiative deexcitation is a quantum process, but $A_{\nu}(E) d \nu$, the probability per unit time of spontaneous emission of a photon in $[\nu, \nu+d \nu]$ by a $\mathrm{PAH}$ with internal energy $E$, can be approximated by

$$
A_{\nu}(E) \approx \frac{4 \pi}{h \nu} C_{\mathrm{abs}}(\nu) B_{\nu}(T(E))
$$

where $B_{\nu}(T)=$ blackbody function, and $T(E)$ is such that an ensemble of such PAHs at temperature $T$ would have average vibrational energy equal to $E$. The thermal approximation is valid except at the lowest vibrational energies (Allamandola et al. 1989; Leger et al. 1989; Draine \& Li 2001).

For small PAHs, the time between photon absorptions is in general long compared to the time required for the PAH to deexcite by IR emission, and therefore the PAH temperature $T$ undergoes large excursions. For larger particles, and intense radiation fields, the grain is unable to fully cool between photon absorptions. The PAH temperature distribution function is found by solving the equations of statistical equilibrium, with upward transitions due to photon absorptions, and downward transitions due to spontaneous emission. Figure 2 shows examples of temperature distribution functions for PAH ions.

The temperature distribution function depends on the PAH size, and therefore the total emitted spectrum will be sensitive to the PAH size distribution $d n / d a$. Figure 3 shows $d n / d a$ adopted by DL07 for diffuse clouds in the MW, as well as that used by Zubko et al. (2004). While both have similar amounts of carbonaceous 


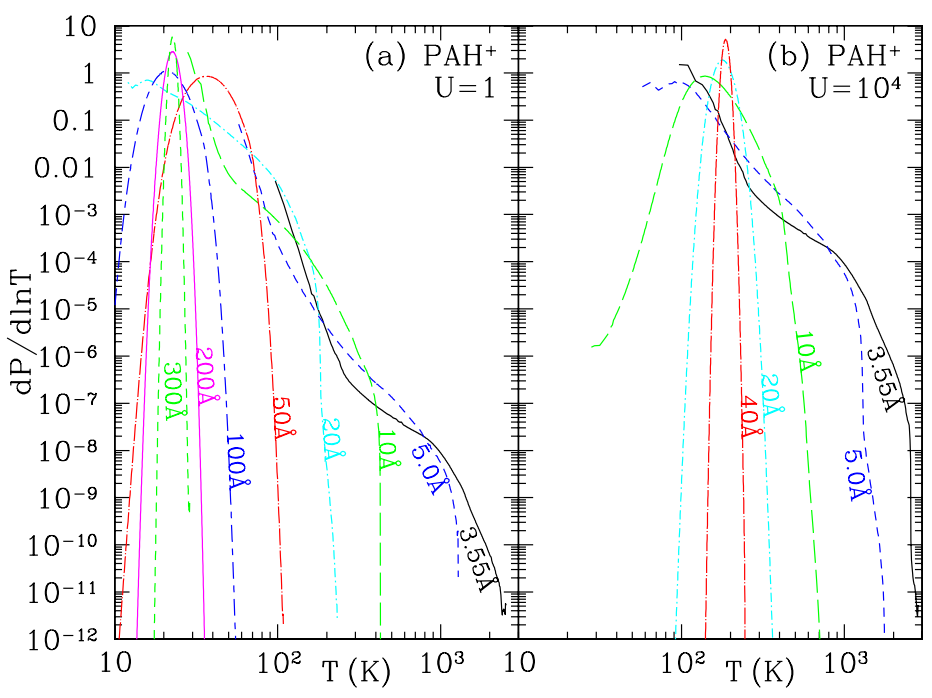

Fig. 2. Temperature distribution functions for $\mathrm{PAH}^{+}$particles in (a) the standard ISRF, and (b) a radiation field $10^{4}$ times more intense than the standard ISRF. Curves are labelled by the radius of an equal-volume sphere. After Draine \& Li (2007).

material in particles with $<10^{3} \mathrm{C}$ atoms, the size distributions differ in detail. The differences can be taken as an indication of the uncertainties.

PAHs will in general be rotationally excited. They are expected to have nonzero electric dipole moments, and, therefore, to emit electric dipole radiation in rotational transitions (see Verstraete 2011 in this volume). Draine \& Lazarian (1998a) proposed that the PAHs could account for the dust-correlated microwave emission discovered by COBE (Kogut et al. 1996). The angular momentum quantum number $J \gtrsim 10^{2}$, allowing a largely classical treatment of the rotational excitation and damping (Draine \& Lazarian 1998b). The rotation rate depends on the PAH size. For the size distribution used to account for the PAH emission features in the infrared results, the predicted rotational emission appears to be in agreement with the observed intensity of dust-correlated microwave emission (e.g., Dobler et al. 2009; Ysard et al. 2010). There do not appear to be any mechanisms that can effectively align PAH angular momenta with the galactic magnetic field (Lazarian \& Draine 2000), and the rotational emission from PAHs is therefore expected to be essentially unpolarized, in agreement with upper limits on the polarization of the dust-correlated microwave emission (Battistelli et al. 2006; Mason et al. 2009). By contrast, the far-infrared and submm emission from larger grains is expected to have polarizations as large as $\sim 10 \%$ (Draine \& Fraisse 2009) - soon to be measured by Planck.

Recent theoretical studies have refined the treatment of the rotational dynamics of small particles (Ali-Haïmoud et al. 2009; Hoang et al. 2010; Silsbee et al. 2010; Ysard \& Verstraete 2010). The evidence to date supports the view that the PAH 


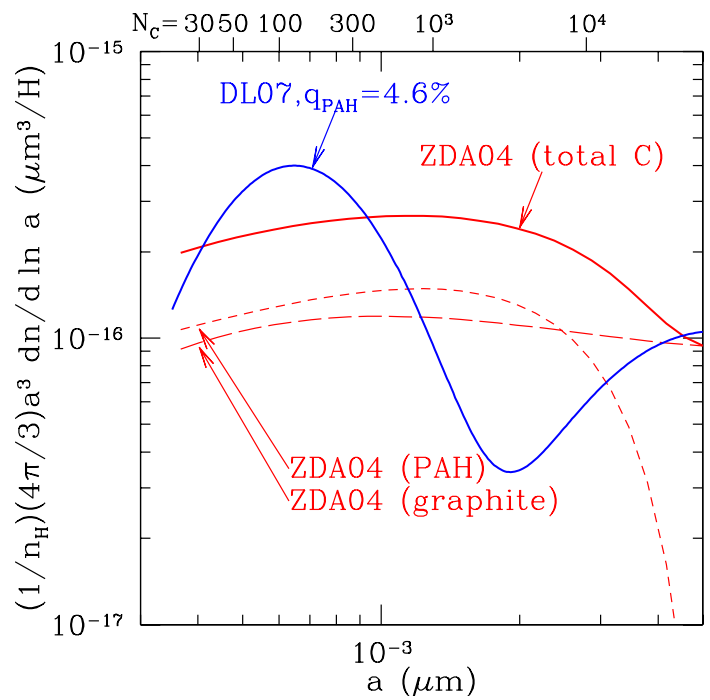

Fig. 3. Volume distributions $\propto a^{3} d n / d \ln a$ for small carbonaceous particles. $a$ is the radius of an equal-volume sphere; the number of carbon atoms $N_{\mathrm{C}}$ is shown at the top of the figure. The curve labeled DL07 is the size distribution used by Draine \& Li (2007); the curves labeled ZDA04 are for graphite and PAH particles from Zubko et al. (2004).

population is responsible for the bulk of the observed dust-correlated microwave emission.

\section{$4 \mathrm{PAH}$ ionization balance}

The PAH charge state changes due to collisions with electrons, collisions with ions, and photoelectric emission. Rates for these processes can be estimated, and the steady-state charge distribution function can be solved for as a function of size and environment. Figure 4 shows the calculated $\phi_{\text {ion }}$, the fraction of PAHs that are non-neutral, as a function of size. The solid curve is a weighted sum over the three idealized environments.

\section{Comparison of model and observed spectra}

The DL07 dust model has been applied to try to reproduce observed IR emission from galaxies. The model is found to reproduce the broad-band photometry from galaxies both globally (e.g., Draine et al. 2007) and within galaxies (e.g., Muñoz-Mateos et al. 2009). How well does the model compare with observed spectra?

Figure 5 shows the low-resolution spectroscopy and broadband photometry measured in the central few kpc of SINGS galaxies (Smith et al. 2007), together 


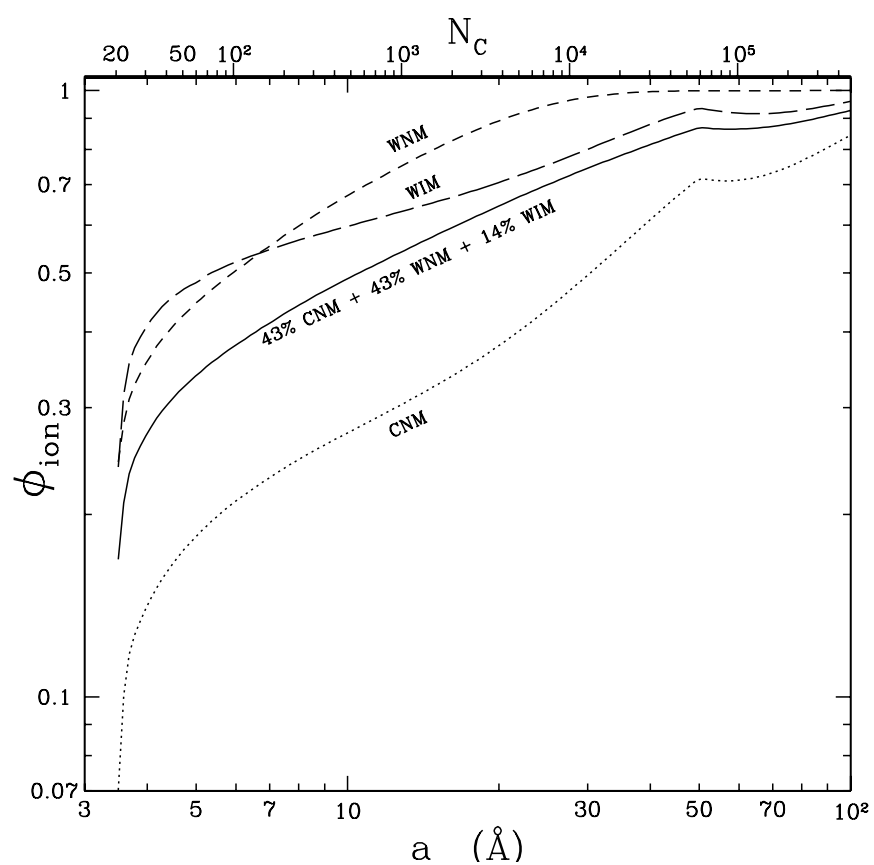

Fig. 4. Calculated ionized fraction $\phi_{\text {ion }}$ for PAHs, as a function of PAH size. Solid curve is $\phi_{\text {ion }}$ adopted by DL07 for the overall ISM. From Li \& Draine (2001a).

with the spectrum of a best-fit DL07 dust model, where the total dust mass, the $\mathrm{PAH}$ abundance (as a fraction of the total grain mass), and the distribution of starlight intensities have been adjusted. The agreement is impressive, particularly since two things that were not varied were (1) the PAH ionization fraction $\phi_{\text {ion }}(a)$ (held fixed at the distribution originally estimated to apply to the Milky Way see Fig. 4), and (2) the spectrum of the starlight heating the dust (taken to be the Mathis et al. 1983, hereafter MMP83) ISRF spectrum.

\section{Spectral variations}

While the overall agreement between model and observations in Figure 5 is excellent, close scrutiny discloses differences. In NGC 5195, the observed ratio of the $7.7 \mu \mathrm{m}$ feature to the $11.3 \mu \mathrm{m}$ feature falls below the model; for NGC 6946 the observed 7.7/11.3 ratio is slightly higher than the model.

A systematic study of variations in PAH spectra was carried out by Galliano et al. (2008). Figure 6a shows examples of variations in the $5-16 \mu \mathrm{m}$ PAH spectra from galaxy to galaxy, and within M 82. The ratio of the power in the $7.7 \mu \mathrm{m}$ complex to the power in the $11.3 \mu \mathrm{m}$ feature varies considerably. Figure $6 \mathrm{~b}$ shows that while $I(7.7) / I(11.3)$ varies by up to a factor $6, I(7.7) / I(6.2)$ and $I(8.6) / I(6.2)$ remain nearly constant. 


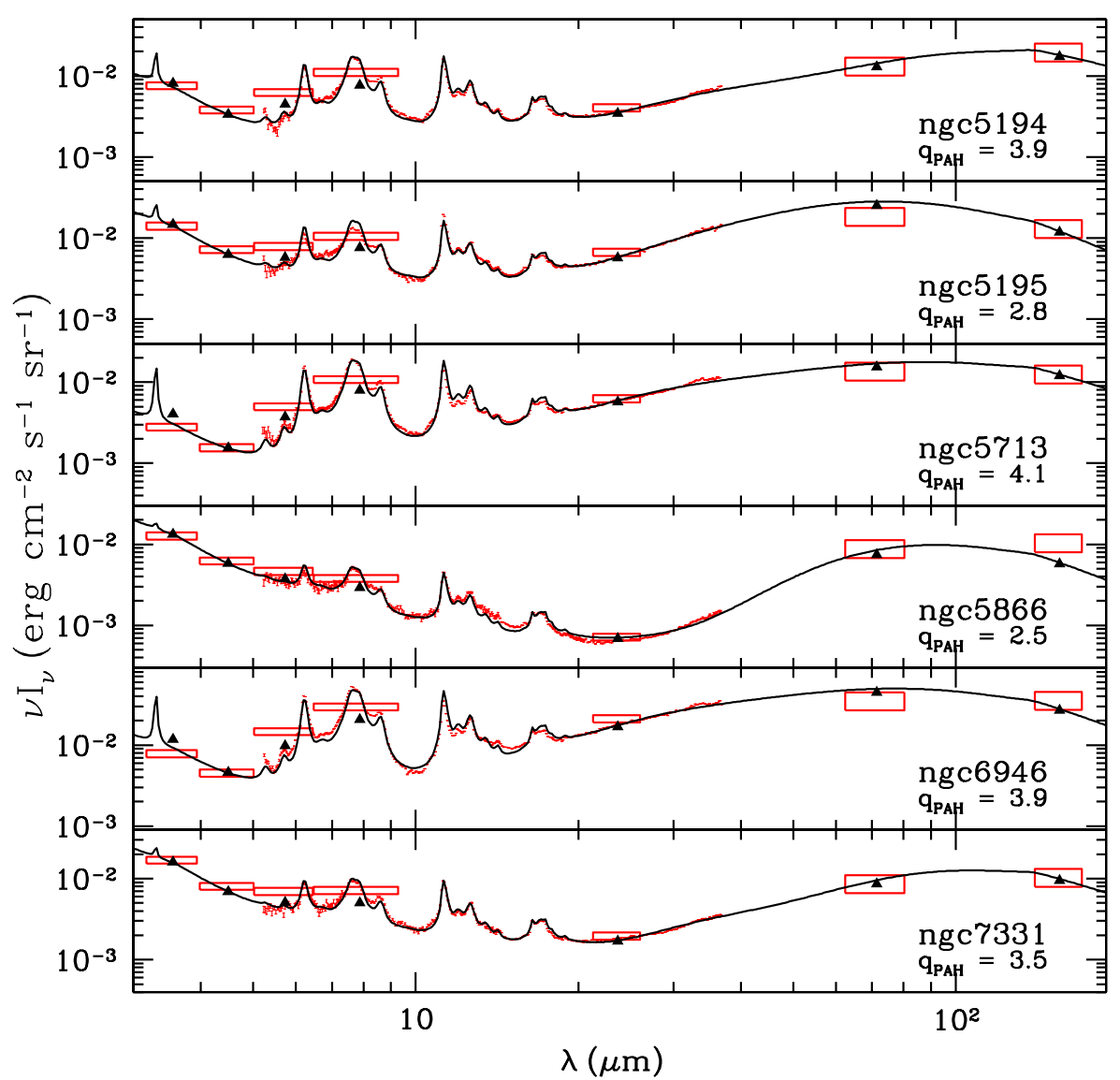

Fig. 5. Spectra of the central regions of SINGS galaxies (Smith et al. 2007), reproduced by models of starlight plus IR emission from dust. Numerous (very small) data points with error bars are $5.3-38 \mu \mathrm{m}$ IRS observations, and the rectangles are IRAC and MIPS photometry for the region where the IRS spectrum was extracted. Solid curves are calculated for the dust model; the triangles show the model convolved with the IRAC and MIPS response functions. For each model the value of $q_{\mathrm{PAH}} \equiv$ (the fraction of total dust mass contributed by PAHs with $N_{\mathrm{C}}<10^{3} \mathrm{C}$ atoms) is given (Reyes et al., in preparation).

Figure $6 \mathrm{c}$ shows that for normal galaxies the $7.7 / 11.3$ ratio does not appear to depend on the hardness of the radiation field (using the NeIII/NeII ratio in H II regions as a proxy). However, very low values of $7.7 / 11.3$ can be found for some AGN with high ratios of $[\mathrm{NeIII}] 15.6 \mu \mathrm{m} /[\mathrm{NeII}] 12.8 \mu \mathrm{m}$.

Can the present dust model accomodate the observed variations in band ratios? Here we consider the effect of changing the PAH ionization, and changing the spectrum of the starlight heating the dust. 


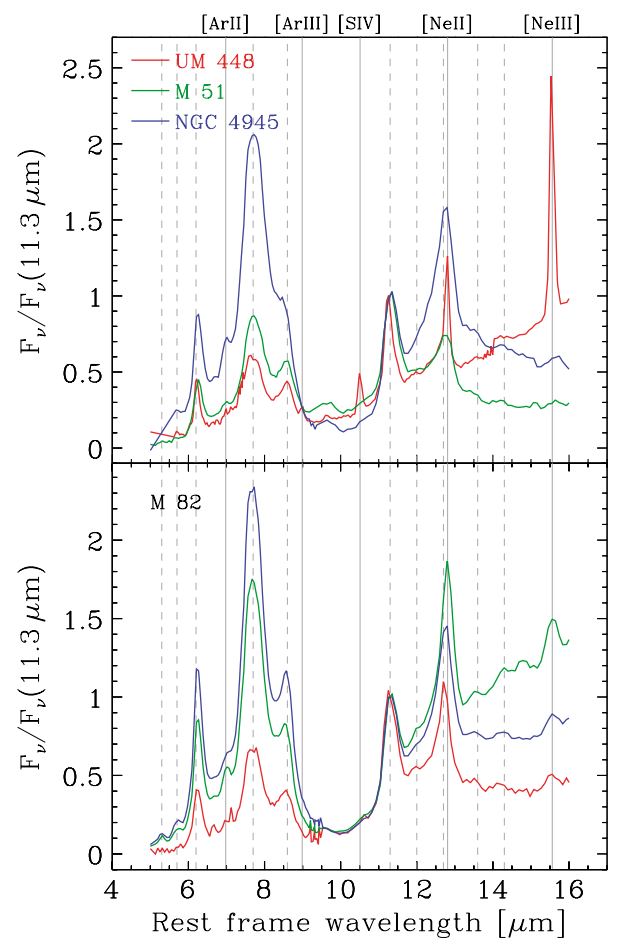

(a) from Galliano et al. (2008)

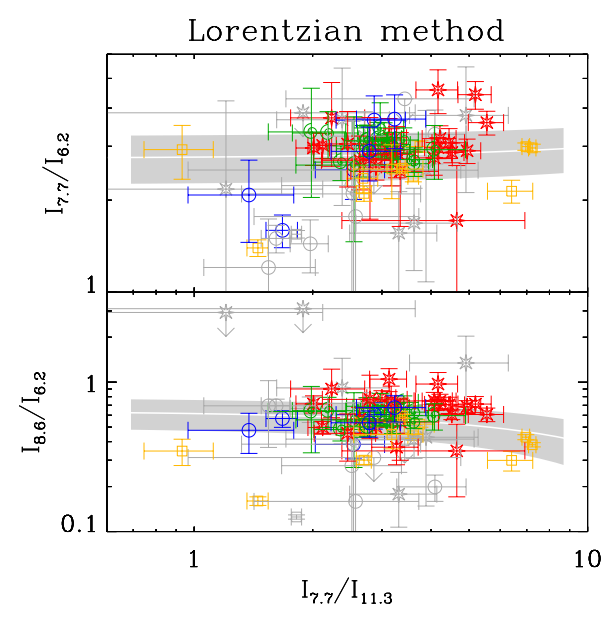

(b) from Galliano et al. (2008)

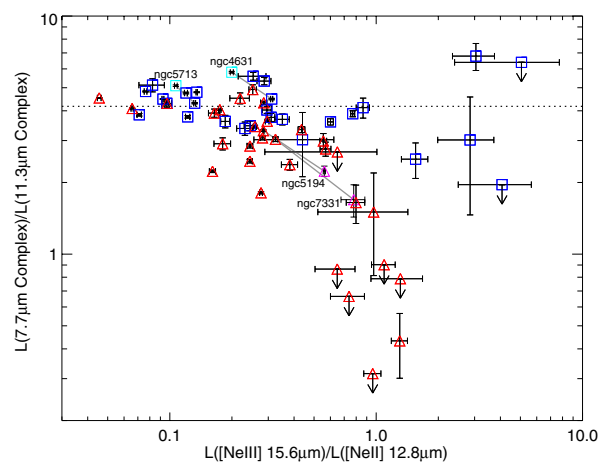

(c) from Smith et al. (2007)

Fig. 6. (a) Variations in spectra from galaxy to galaxy, and within M 82 (from Galliano et al. 2008). (b) In a sample of 50 objects, the $I(7.7 \mu \mathrm{m}) / I(11.3 \mu \mathrm{m})$ ratio varies by a factor $\sim 6$, while $I(7.7) / I(6.2)$ and $I(8.6) / I(6.2)$ remain relatively constant (from Galliano et al. 2008). (c) Variations of $I(7.7) / I(11.3)$ among galaxies, as a function of the $[\mathrm{NeIII}] 15.6 \mu \mathrm{m} /[\mathrm{NeII}] 12.8 \mu \mathrm{m}$ flux ratio. Squares: normal HII-type galaxies; Triangles: AGN (from Smith et al. 2007).

\section{PAH ionization}

With the ionization-dependent opacities of Figure 1, we can alter the emission spectrum by changing the adopted ionization function $\phi_{\text {ion }}(a)$. Figure 7 a shows emission spectra calculated for two extreme assumptions regarding the ionization: $\phi_{\text {ion }}=0$ and $\phi_{\text {ion }}=1$, as well as for $\phi_{\text {ion }}$ adopted by DL07 (shown in Fig. 4). It is apparent that the $7.7 / 11.3$ band ratio increases considerably as the PAH 

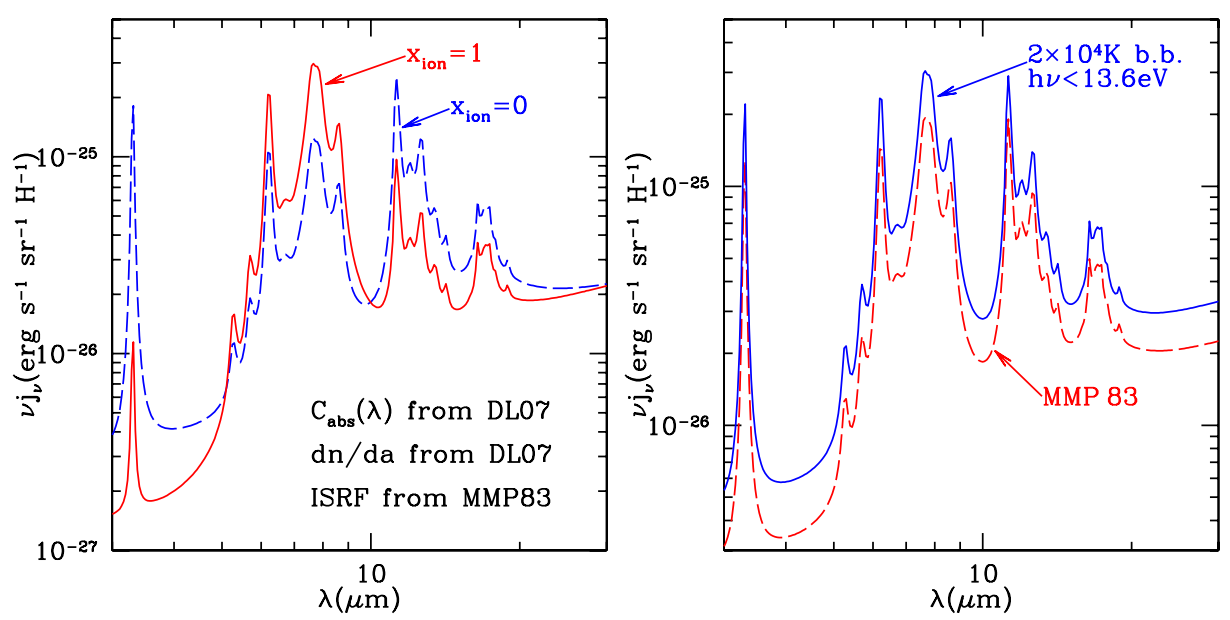

Fig. 7. (a) 3-30 $\mu \mathrm{m}$ emission spectrum for fully-ionized (solid curve) and neutral PAHs (broken curve) heated by the local ISRF (Mathis et al. 1983, MMP83). The 7.7/11.3 band ratio changes by a factor $\sim 6$. (b) 3-30 $\mu$ m emission spectrum for fixed ionization $\phi(a)$ used by DL07, but comparing PAHs heated by the MMP83 ISRF (broken curve) vs. PAHs heated by a $2 \times 10^{4} \mathrm{~K}$ blackbody cut off at $13.6 \mathrm{eV}$ (solid curve). The $7.7 / 11.3$ band ratio is essentially unchanged.

Table 1. PAH band ratios calculated for the DL07 model.

\begin{tabular}{cccc}
\hline$\phi_{\text {ion }}$ & Radiation Field & $\left(\nu P_{\nu}\right)_{7.7 \mu \mathrm{m}} /\left(\nu P_{\nu}\right)_{11.3 \mu \mathrm{m}}$ & $\left(\nu P_{\nu}\right)_{7.7 \mu \mathrm{m}} / P(\mathrm{TIR})^{a}$ \\
\hline$\phi_{\text {ion }}=0$ & MMP83 & 0.487 & 0.323 \\
$\phi_{\text {ion }}=0$ & 20 kK blackbody & 0.512 & 0.523 \\
DL07 & MMP83 & 1.01 & 0.499 \\
DL07 & 20 kK blackbody & 1.05 & 0.783 \\
$\phi_{\text {ion }}=1$ & MMP83 & 3.08 & 0.759 \\
$\phi_{\text {ion }}=1$ & $20 \mathrm{kK}$ blackbody & 3.36 & 1.162 \\
\hline
\end{tabular}

${ }^{a} P(\mathrm{TIR})=\int P_{\nu} d \nu$ is the total IR power for the dust model.

ionized fraction is increased. Table 1 shows that for the adopted $\mathrm{PAH}^{0}$ and $\mathrm{PAH}^{+}$ absorption cross sections (see Fig. 1) the 7.7/11.3 feature ratio changes by a factor $\sim 6.3$ as the ionization is varied - a range comparable to what is actually seen (see Fig. 6b)!

\section{Variations in starlight spectrum?}

There will naturally be variations in the spectrum of the starlight heating PAHs, both regionally within a galaxy, and from one galaxy to another. Heating by a harder radiation field will lead to an increase in the fraction of the dust heating contributed by hard photons, and therefore will increase the short wavelength PAH emission. However, Galliano et al. (2008) showed that the harder starlight 
spectrum of a starburst does not increase the $7.7 / 11.3$ ratio by enough to explain the observed variations. Figure 7 compares the $3-30 \mu \mathrm{m}$ emission spectrum for PAHs heated by the MMP83 ISRF, vs. dust heated by a $2 \times 10^{4} \mathrm{~K}$ blackbody cut off at $13.6 \mathrm{eV}$. The $2 \times 10^{4} \mathrm{~K}$ blackbody is adjusted in intensity to give the same overall power per $\mathrm{H}$ absorbed by dust. The harder radiation field does lead to an increase in the PAH emission as a fraction of the total, but has only a small effect on the $7.7 / 11.3$ band ratio (only a $4 \%$ change - see Table 1 ), confirming the finding of Galliano et al. (2008).

\section{Changes in the PAH size distribution?}

What about the lowest values of 7.7/11.3 found for some AGN (see Fig. 6c)? The smallest PAHs may be susceptible to destruction by X-rays Voit (1992). Suppression of the abundances of PAHs with $<10^{3} \mathrm{C}$ atoms (while leaving larger PAHs unchanged) would reduce the $7.7 / 11.3$ ratio, lowering it by a factor $\sim 1.5$ (Galliano et al. 2008); however, as noted by Galliano et al. (2008), this change to the size distribution would also lower the $6.2 / 7.7$ band ratio, which does not appear to vary in normal galaxies; hence this effect would apply only in unusual objects.

\section{What is the relation between PAHs and the $2175 \AA$ A feature?}

The strength of the interstellar $2175 \AA$ feature requires that the carrier $X$ have $n_{X} f_{X} / n_{\mathrm{H}} \approx 9.3 \times 10^{-6}$ (Draine 1989), where $f_{X}$ is the oscillator strength per $X$. In general, PAHs have strong absorption near $2200 \AA$ due to $\pi \rightarrow \pi^{*}$ electronic excitation. For small graphite spheres, the $\pi \rightarrow \pi^{*}$ excitation has an oscillator strength $f \approx 0.16$ per $\mathrm{C}$ atom. If we assume a similar oscillator strength for the $\pi \rightarrow \pi^{*}$ transition in PAHs, then $\mathrm{C} / \mathrm{H} \approx 58 \mathrm{ppm}$ in PAHs would be sufficient to account for the observed integrated strength of the $2175 \AA$ feature. The abundance of PAHs required to explain the observed IR emission is 30-60 ppm ( $\mathrm{Li} \&$ Draine 2001a; Draine \& Li 2007). Therefore, it seems likely that the $2175 \AA$ feature is due mainly to PAHs (Joblin et al. 1992; Li \& Draine 2001a).

The $s p^{2}$-bonded carbon in PAHs also has strong absorption in $\sigma \rightarrow \sigma^{*}$ transitions, resulting in absorption rising steeply beginning at $\sim 1200 \AA(\sim 10 \mathrm{eV})$ and peaking at $\sim 720 \AA(\sim 17 \mathrm{eV})$. Thus, any sightline with $2175 \AA$ absorption (if due to PAHs) should also have steeply rising extinction at $\lambda \lesssim 1200 \AA$, although other elements of the dust population (e.g., small silicate particles) can also contribute steeply rising far-UV extinction in addition to that provided by the PAHs.

PAH emission features are ubiquitous in IR spectra of star-forming galaxies, including starburst galaxies. However, the $2175 \AA$ feature is weak or absent in spectra of starburst galaxies: the "Calzetti extinction law" for these starbursts shows no $2175 \AA$ feature (Calzetti et al. 1994). Conroy (2010) found no evidence of $2175 \AA$ extinction in the spectra of star-forming galaxies at $z \approx 1$. Note, however, that Conroy et al. (2010) do find evidence for $2175 \AA$ extinction in GALEX observations of nearby galaxies. Given the ubiquity of PAH emission in star-forming galaxies, the weakness or absence of the $2175 \AA$ feature in the spectra of starburst galaxies, and in the $z \sim 1$ sample of Conroy (2010), is surprising. 
Absence of the $2175 \AA$ feature in starburst spectra may be in part the result of complex geometry and radiative transfer - perhaps the regions in the galaxy that dominate the emergent FUV may be regions where the PAHs have been destroyed.

In the SMC, 4/5 sightlines where UV extinction has been measured have no detectable $2175 \AA$ feature (Gordon et al. 2003). If PAHs absorb near $2175 \AA$, then we expect PAH emission to be weak in the SMC. From COBE-DIRBE observations, Li \& Draine (2002) argued that PAH emission was strongly suppressed (relative to total IR emission) in SMC (but this was controversial - see Bot et al. 2004).

Recently, Sandstrom et al. (2010) studied PAH emission in the SMC, finding that the PAH emission, while not zero, is indeed weak, consistent with the $2175 \AA$ extinction feature being due to PAHs. Sandstrom et al. also found large regional variations in the $\mathrm{PAH}$ abundance within the $\mathrm{SMC}$, with the $\mathrm{PAH}$ abundance peaking near molecular clouds.

\section{Summary}

The principal points are the following:

- Physical models for interstellar dust can reproduce the observed PAH emission features together with other observed properties, including microwave emission, submm and far-IR emission, and extinction and scattering from the near-IR to X-ray energies. Amorphous silicate material accounts for $\sim 75 \%$ of the dust mass. Carbonaceous material accounts for $\sim 25 \%$ of the mass, with $\sim 5 \%$ of the total dust mass in PAHs.

- Lacking detailed knowledge of the physical properties of PAHs, current models use very simplified representations of PAH absorption as a function of wavelength. At present these properties are "tuned" to be able to reproduce astronomical observations, but the adopted opacities appear to be consistent with what is known of PAHs in the IR through ultraviolet.

- PAH band ratios are observed to vary from region to region. The 7.7/11.3 band ratio varies by up to a factor $\sim 6$. Model calculations (see Fig. 7) show that this could result from changes in the PAH ionization balance from nearly fully neutral (low 7.7/11.3 ratio) to nearly fully ionized (high $7.7 / 11.3$ ratio).

- The $2175 \AA$ interstellar extinction feature should be due, at least in part, to $\pi \rightarrow \pi^{*}$ absorption in PAHs. Weakness or absence of the $2175 \AA$ feature would then imply weakness or absence of PAH emission. The $2175 \AA$ feature is observed to be weak in the SMC; recent study of the PAH emission from the SMC confirms that, as predicted, the PAH abundance is very low (Sandstrom et al. 2010).

- Existing models use highly simplified descriptions of the PAH absorption cross sections from the UV to the IR, with considerable freedom for adjustment 
to reproduce astronomical observations. As both laboratory and theoretical understanding moves forward, modeling will become more strongly constrained.

I am grateful to Christine Joblin and the SOC for organizing this meeting, and for the invitation to speak at it. I thank Xander Tielens for comments and suggestions that helped improve this text. This work was supported in part by NASA through JPL grant 1329088 .

\section{References}

Ali-Haïmoud, Y., Hirata, C.M., \& Dickinson, C., 2009, MNRAS, 395, 1055

Allamandola, L.J., Tielens, G.G.M., \& Barker, J.R., 1989, ApJS, 71, 733

Battistelli, E.S., Rebolo, R., Rubiño-Martín, J.A., et al., 2006, ApJ, 645, L141

Bot, C., Boulanger, F., Lagache, G., Cambrésy, L., \& Egret, D., 2004, A\&A, 423, 567

Calzetti, D., Kinney, A.L., \& Storchi-Bergmann, T., 1994, ApJ, 429, 582

Cecchi-Pestellini, C., Malloci, G., Mulas, G., Joblin, C., et al., 2008, A\&A, 486, L25

Compiegne, M., Verstraete, L., Jones, A., et al., 2010 [ArXiv 1010.2769v1]

Conroy, C., 2010, MNRAS, 404, 247

Conroy, C., Schiminovich, D., \& Blanton, M.R., 2010, ApJ, 718, 184

Desert, F.-X., Boulanger, F., \& Puget, J.L., 1990, A\&A, 237, 215

Dobler, G., Draine, B., \& Finkbeiner, D.P., 2009, ApJ, 699, 1374

Draine, B.T., 1989, ed. L. Allamandola \& A. Tielens, IAU Symp. 135: Interstellar Dust (Kluwer, Dordrecht), 313

Draine, B.T., 2003, ARAA, 41, 241

Draine, B.T., Dale, D.A., Bendo, G., et al., 2007, ApJ, 663, 866

Draine, B.T., \& Fraisse, A.A., 2009, ApJ, 696, 1

Draine, B.T., \& Lazarian, A., 1998a, ApJ, 494, L19

Draine, B.T., \& Lazarian, A., 1998b, ApJ, 508, 157

Draine, B.T., Li, A., 2001, ApJ, 551, 807

Draine, B.T., \& Li, A., 2007, ApJ, 657, 810

Galliano, F., Madden, S.C., Tielens, A.G.G.M., Peeters, E., \& Jones, A.P., 2008, ApJ, 679,310

Gordon, K.D., Clayton, G.C., Misselt, K.A., Landolt, A.U., \& Wolff, M.J., 2003, ApJ, 594,279

Hoang, T., Draine, B.T., \& Lazarian, A., 2010, ApJ, 715, 1462

Joblin, C., Leger, A., and Martin, P., 1992, ApJ, 393, L79

Kogut, A., Banday, A.J., Bennett, C.L., et al., 1996, ApJ, 464, L5

Lazarian, A., \& Draine, B.T., 2000, ApJ, 536, L15

Leger, A., D’Hendecourt, L., \& Defourneau, D., 1989, A\&A, 216, 148

Li, A., \& Draine, B.T., 2001a, ApJ, 554, 778

Li, A., \& Draine, B.T., 2001b, ApJ, 550, L213

Li, A., \& Draine, B.T., 2002, ApJ, 576, 762

Mason, B.S., Robishaw, T., Heiles, C., Finkbeiner, D., \& Dickinson, C., 2009, ApJ, 697, 1187 
Mathis, J.S., Mezger, P.G., \& Panagia, N., 1983, A\&A, 128, 212

Muñoz-Mateos, J.C., Gil de Paz, A., Boissier, S., et al., 2009, ApJ, 701, 1965

Sandstrom, K.M., Bolatto, A.D., Draine, B.T., Bot, C., \& Stanimirovic, S., 2010, ApJ, 715,701

Silsbee, K., Ali-Haimoud, Y., \& Hirata, C.M., 2010 [arXiv1003.4732]

Smith, J.D.T., Draine, B.T., Dale, D.A., et al., 2007, ApJ, 656, 770

Tielens, A.G.G.M., 2008, ARAA, 46, 289

Voit, G.M., 1992, MNRAS, 258, 841

Weingartner, J.C., \& Draine, B.T., 2001, ApJ, 548, 296

Ysard, N., Miville-Deschênes, M.A., \& Verstraete, L., 2010, A\&A, 509, L1

Ysard, N., \& Verstraete, L., 2010, A\&A, 509, A12

Zubko, V., Dwek, E., \& Arendt, R.G., 2004, ApJS, 152, 211 